Animal Health Research Institute, El-Mansoura Lab.

\title{
FISH AS A MIRROR FOR WATER POLLUTION
}

(With 10 Tables)

By

\section{AZZA E.A. HASSAN and RAWIA S.M. EL-ADAWI \\ (Received at 5/3/2011)}

الأسماك كمرآه لتثلوث المياه

\section{عزة السبي على حسان ، راوية سعد محمد العدوي}

أجريت هذه الدر اسة بهدف معر فة تلوث المياه وانعكاس هذا التلوث على الأسماك النيلية. فقد 55 الند

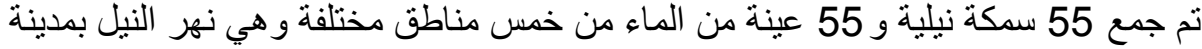

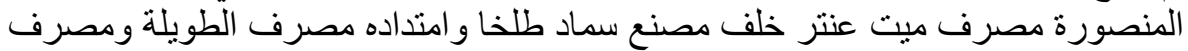

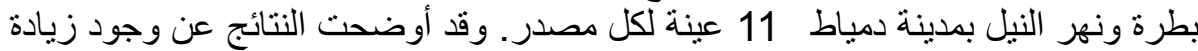

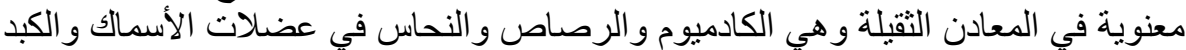

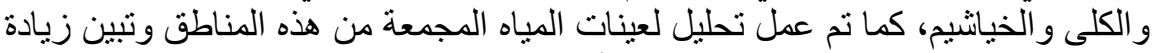

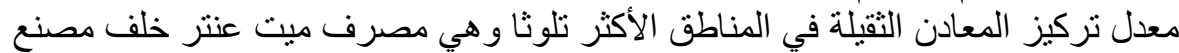

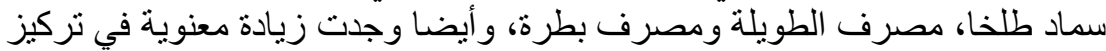

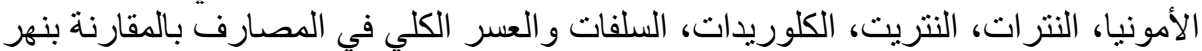

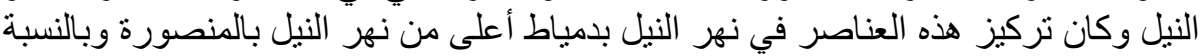
للماثثيون اوضحت النتائج أن الملاثيون وجد في جميع المناطق ماعدا نهر النئ النيل بمدينة

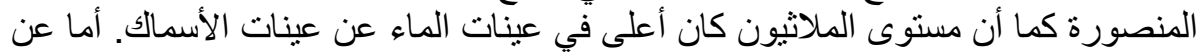

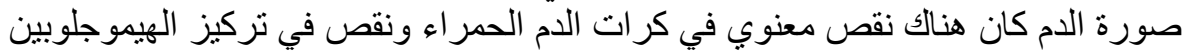

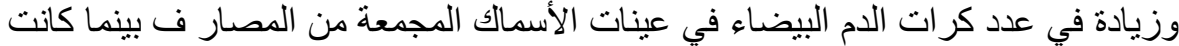

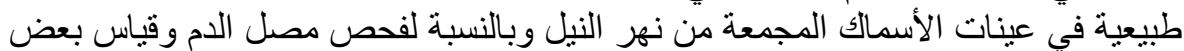

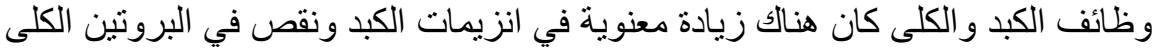

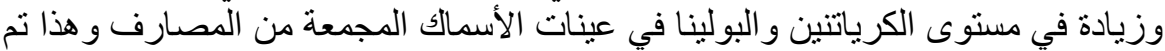
تأكيده بقياس جهاز الجلوتاثيون، وقد كان هنالك نقص معنوي في الجلوناثيون الكلي والثان المختزل

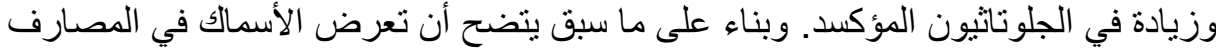
للملوثات بؤدي إلى تغير ات سلبية كبيرة وتحوير في جهاز الجلوتاثيون إلى النى الناحية التأكسدية.

\section{SUMMARY}

One hundred ten samples from water and tilapia fish from 5 different location, River Nile Mansoura, Met-Annter drainage behind Talkha factory of fertilization, El-Tawilla drainage, Battra drainage and River 
Nile Damietta were collected for analysis of heavy metals, malathion in water and fish also analysis of water chemical parameters ammonia, nitrite, nitrate, chlorides, sulphates and total hardness, analysis hepatic glutathione system, some liver and kidney functions and blood picture The results showed highly significant increase of heavy metals and malathion in both fish and, water samples were collected from MetAnnter, El-Tawilla and Battra drainages. Malathion concentration in water was higher than in fish samples regarding to water chemical parameters ammonia, nitrite, nitrate, chlorides, sulphates and total hardness showed highly significant increase in drainages comparing with River Nile, and the presence of these chemicals parameters was higher in River Nile Damietta than in River Nile Mansoura. Meanwhile the results showed shifting of the glutathione system manifested by dropping of total and reduced glutathione in hepatic tissue and increasing of oxidized glutathione in hepatic tissue. Moreover, showed significant increase in serum ALT, AST, creatinine and blood urea, while samples collected from River Nile in Mansoura were within normal level. Concerning to the red blood cell count (RBCs), heamoglobin $(\mathrm{Hb})$ and packed cell volume (PCV) levels were lower than fish collected from River Nile at Mansoura which were in normal ranges. The total leukocytes count were in normal range in fish collected from River Nile Mansoura, in contaray total leukocytic count were increased in other polluted areas. Meanwhile shifting of glutathione system towards the oxidized side pointed to hepatotxic and nephrotoxic as these pollutants consider as an oxidant. The aim of this study was to know the water pollution and the reflection of this pollution on fish. The study concluded that the heavy metals and other pollutants have a very dangerous effect on biochemical parameters and vital organs of fish.

Key words: Fish, water pollution, heavy metals, blood picture.

\section{INTRODUCTION}

Environmental pollution represents a major problem in the world, especially in the developed countries. Egypt is one of such countries, which suffers from biospheres pollution air and water pollution (Mona and Sawsan, 2004).

All water contains natural contaminations particularly inorganic that arise from the geological strata through which the water flows and 
to a varying extent anthropogenic pollution by chemicals (Fawell and Nieuwenhuijsen, 2003).

Heavy metals have a great ecological significance due to their toxicity and a cumulative behavior playing a role with aquatic organisms, therefore; the toxic heavy metals become a hazard for mammals De (Gregori et al., 1994). Heavy metals are divided into non essential metals, which have no role in the biological processes in human or animal, as lead, cadmium and mercury, while essential metals are needed for human and animal body consumption, are like copper, zinc, manganese and cobalt (Moalla and Pulford, 1995; Gold Frank et al., 2001).

Several sources of the environmental pollution of heavy metal including the industrial and agricultural discharge, coal and oil combustion, sewage effluents, some types of plastics, pesticides, lead, cadmium and mercury pollutions in fish and natural sources from rocks and soil (WHO, 1992; Shibamoto and Bjeldanes, 1993; Jehan et al., 1999; Abd-El-Hamid et al., 2006).

Pesticides as malathions constitutes an important aspect of modern agriculture, the public has been tolerant of their use Aspelin (1998). 85-90\% of the pesticides applied in agriculture never reaches their target organisms, but instead are dispersed in the air, water and soil (Repetto and Baliga, 1996).

One of the most serious problems is due to the runoff and drainage in land escapes where pesticides are applied. This may carry residues into drinking water supplies where they are dissolved or lie suspended in water. This, results in the pollution of water where by the quality of the water deteriorates and threatens aquatic ecosystem health (Wakawa, 2008)

Pollution of the environment is either by improper application or due to pesticides persistence in soil, plants, water and animal tissue when these substances pollute lakes, streams, rivers and other water bodies. Malathion is a non-systemic wide spectrum organophosrus insecticide (USEPA, 2000).

Fish occupy one of the foremost places among the food products of animal origin in their nutritive value and this may be attributed to their contents of high biological value proteins, lipids, vitamins and essential fatty acids (Darwish et al., 2003; Burger et al., 2005) as well as it provides omega-3 fatty acids, which have many health benefits (Patterson, 2002). 
Heavy metals in surface water may be run-off from land application, dumped from domestic and industrial sewage, atmospheric deposition of mining practices, improper handling of mining tailings, or as results of corrosion of distribution system materials (Calderon, 2000). Fish are continuously exposed to water, the major sink for many contaminations, such as anthropogenic and other contaminants always impact fish causing serious hazards (Denslow et al., 2007). Therefore, the aim of this work is to detecte heavy metal, ammonia, nitrite, nitrates, chlorides, sulphates in water, and malathion in water and fish, some liver and kidney functions, blood parameters, reduced glutathione and total glutathione were also estimated.

\section{MATERIALS and METHODS}

\section{Sampling}

\section{Water}

- A total of fifty five water samples were collected from five different regions in Dakahlia Governorate from River Nile in Mansoura, Met Annter Drainage behind the fertilization factory of Talkha, Tawilla drainage, Bettra drainage and from River Nile in Domiitta City. Eleven samples were collected from each region

- The technique of water sampling was carried out according to $\mathrm{HACH}$ (2003) for determination of ammonia, nitrite, nitrate, chlorides, sulphates and malathion.

\section{Fish}

- Fifty five Tilapia fish were collected from five different regions from River Nile in Mansoura, Met Annter Drainage behind the fertilization factory of Talkha, El-Tawilla, Battra drainages and River Nile Damietta

- The collected fishes were placed in polyethylene bag and transported to the laboratory in ice box. The tissue samples from liver, kidney and muscles were taken from the dissected fish and stored in the deep freezer at $-20 \mathrm{C}^{\mathrm{o}}$ for heavy metals evaluation.

\section{Blood}

- Whole blood: About 0.5-1 ml whole blood was collected in small plastic vials containing a suitable amount of dipotassium salt of ethylene Diamine Tetra Acetic acid (EDTA) as anticoagulant. Samples were examined for evaluation of blood picture (Total leucocytic count (TLC), hemoglobin ( $\mathrm{Hb}$ ). Total Erythrocytic count (TEC) and packed cell volume (PCV) according to Stoskopf (1993). 
- Blood serum: About $2 \mathrm{ml}$ blood were collected in plastic centrifuge tube and left to clot at $4 \mathrm{C}^{0}$ after that, it placed to be centrifuged to separate the blood serum. The separated serum was used in biochemical determination of serum alanine aminotransferase (ALT) Young, (2001), serum aspartate aminotransferase (AST) Reitman and Frankel, (1957), serum total protein was measured according to Doumas et al. (1981), blood urea according to Patton and Crouch, (1977) and creatinine (Husden and Ropaport, 1968).

\section{Chemical examination}

- Determination of Ammonia $\left(\mathrm{NH}_{3}\right)$

Ammonia concentration in examined water samples was determined by using "The Direct Nesslerization Method" previously described by APHA, (1985).

- Determination of Nitrite $\left(\mathrm{NO}_{2}\right)$

Level of nitrite in the examined water sample was estimated by APHA, (1998).

- Determination of Nitrate $\left(\mathrm{NO}_{3}\right)$

Concentration of nitrates in the examined water sample was determined by "Brucine Method" according to APHA (1960).

- Determination of Chlorides $\left(\mathrm{Cl}_{2}\right)$

Chlorides in examined water sample were estimated by "Argentometric Method" described by (APHA, 1998).

- Determination of sulphate (SO4)

Concentration of sulphate in the examined water sample was determined by APHA (1998).

- Determination of heavy metals

Preparation of examined water samples was carried out according to APHA (1985), where the water sample was filtered through $0.45 \mu$ what man filter. The required volume $100 \mathrm{ml}$ of filtrate was collected in clean glass bottle. Preserved by $0.3 \mathrm{ml}$ of nitric acid and kept in refrigerator. Determination of copper $(\mathrm{Cu})$, lead $(\mathrm{Pb})$ and cadmium (Cd) in surface water was carried according to the methods prepared by Polpraset (1982); Sprenger et al. (1987).

- Determination of malathion

Samples were prepared for malthion detection according to the method described by Jadhav et al. (1992).

- Determination of Copper, Cadmium and lead

Samples were taken from fish tissues, and then were digested according to the method described by Heckman (1970); Greig et al. 
(1982). Tissue samples were measured by using atomic absorption spectrophotometer according to Capar (1977).

- Determination of hepatic reduced Glutathione

The amount of GSH in the supernatant obtained after the centrifugation of liver homogenate at $3000 \mathrm{rpm}$ for 30 minutes was determined by using the method of Richardson and Murphy (1987).

- Determination of hepatic total Glutathione (GSH and GSSH)

Total Glutathion was determined enzymatically according to DTNBGSSG reductase recycling assay for GSH and GSSG as described by Anderson (1985).

- Determination of hepatic oxidized Glutathione

Oxidized glutathione (GSSG) was determined after the reduced glutathione concentration was subscripted from the total glutathione concentration.

- Statistical analysis of variance (ANOVA) and t-test was carried out following the method described by Kirkwood (1989).

\section{RESULTS}

The present data revealed that fish samples collected from polluted regions. It were significantly high heavy metal concentration comparing with samples collected from River Nile Mansoura, as shown in Tables 2, 3, 4 and 5. Regarding to chemical constitutes of ammonia, nitrate, nitrite,sulphate, chloride, total hardness collected from polluted regions our results showed highly significant increase $(\mathrm{P}<0.001)$ Table 6. Malathion was detected in all examined water and fish samples from different locations except River Nile Mansoura. $\mathrm{P}<0.001$ as shown in Table 7. Concerning blood parameters; results revealed significant decrease in total RBCs, haemoglobin and packed cell volume, while there was highly significant increase in WBCs $(\mathrm{P}<0.001)$ as showen in Table 8. Liver and kidney functions tests revealed significant decrease of total protein, increase of ALT, AST, creatinin and blood urea $\mathrm{P}<0.01$, $\mathrm{P}<0.05$ and $\mathrm{P}<0.001$ respectively Table 9 . Total glutathione and reduced glutathione analysis in fish hepatic tissue revealed significant decrease, $(\mathrm{P}<0.05, \mathrm{P}<0.001$ and $\mathrm{P}<0.01$, $)$ while oxidized glutathione showed significant increase $(\mathrm{P}<0.05)$ as shown in Table 10. 
Table 1: Physical analysis of water and heavy metals concentration in samples of water collected from different locations.

\begin{tabular}{|c|c|c|c|c|c|c|c|}
\hline parameter & $\begin{array}{c}\text { Temperature } \\
\mathrm{C}^{0}\end{array}$ & $\begin{array}{c}\text { Oxygen } \\
\mathrm{ppm}\end{array}$ & $\begin{array}{c}\mathrm{pH} \\
\text { value }\end{array}$ & $\begin{array}{c}\text { Salinity } \\
\mathrm{g} / \mathrm{l}\end{array}$ & $\begin{array}{c}\text { Cadmium } \\
(\mathrm{Cd}) \mathrm{PPm}\end{array}$ & $\begin{array}{c}\text { Lead } \\
\mathrm{Pb} \mathrm{PPm}\end{array}$ & $\begin{array}{c}\text { Copper } \\
(\mathrm{Cu}) \mathrm{PPm}\end{array}$ \\
\hline River Nile (Mansoura) & $23 \pm 2$ & $8.15 \pm 0.61$ & $7.25 \pm 0.42$ & $0.15 \pm 0.02$ & $\begin{array}{c}0.048 \\
\pm 0.006\end{array}$ & $\begin{array}{c}0.009 \\
\pm 0.0003\end{array}$ & $\begin{array}{c}0.067 \\
\pm 0.005\end{array}$ \\
\hline Met-Annter drainage & $24 \pm 2$ & $5.9 \pm 0.24$ & $9.12 \pm 0.83$ & $1.325 \pm 0.08$ & $\begin{array}{c}0.098 \\
\pm 0.006^{* * *}\end{array}$ & $\begin{array}{c}0.89 \\
\pm 0.04^{* * *}\end{array}$ & $\begin{array}{c}1.48 \\
\pm 0.22^{* * *}\end{array}$ \\
\hline Tawilla drainage & $22 \pm 2$ & $6.5 \pm 0.35$ & $8.11 \pm 0.52$ & $1.162 \pm 0.04$ & $\begin{array}{c}0.065 \\
\pm 0.003^{*}\end{array}$ & $\begin{array}{c}0.26 \\
\pm 0.06^{* *}\end{array}$ & $\begin{array}{c}0.92 \\
\pm 0.07^{* * *}\end{array}$ \\
\hline Battra drainage & $22 \pm 2$ & $7.2 \pm 0.38$ & $7.85 \pm 0.49$ & $0.973 \pm 0.06$ & $\begin{array}{c}0.078 \\
\pm 0.004^{* *}\end{array}$ & $\begin{array}{c}0.089 \\
\pm 0.005^{*}\end{array}$ & $\begin{array}{c}0.75 \\
\pm 0.06^{* *}\end{array}$ \\
\hline River Nile Damietta & $21 \pm 2$ & $7.9 \pm 0.42$ & $7.65 \pm 0.53$ & $0.646 \pm 0.03$ & $\begin{array}{c}0.082 \\
\pm 0.005^{* *}\end{array}$ & $\begin{array}{c}0.065 \\
\pm 0.003^{*}\end{array}$ & $\begin{array}{c}0.83 \\
\pm 0.06^{* * *}\end{array}$ \\
\hline
\end{tabular}

${ }^{* * *} \mathrm{P}<0.001 \quad{ }^{* *} \mathrm{P}<0.01 \quad \mathrm{P}<0.05$

Permissible limit of heavy metals in water according to WHO (1993) Cadmium $0.003 \mathrm{mg} / 1$, Lead $0.01 \mathrm{mg} / \mathrm{l}$ Copper $2 \mathrm{mg} / \mathrm{l}$

Table 2: Mean level of heavy metal concentration in organs of Nile Tilapia collected from Met-Annter drainage comparing with samples collected from River Nile Mansoura.

\begin{tabular}{|c|c|c|c|c|c|c|c|c|}
\hline \multirow{2}{*}{$\begin{array}{l}\text { organ } \\
\text { heavy meta }\end{array}$} & \multicolumn{4}{|c|}{$\begin{array}{c}\text { Samples of fish organs collected from } \\
\text { River Nile Mansoura }\end{array}$} & \multicolumn{4}{|c|}{$\begin{array}{c}\text { Samples of fish organs collected from } \\
\text { Met-Annter drainage }\end{array}$} \\
\hline & Muscle & Liver & Kidney & Gills & Muscle & Liver & Kidney & Gills \\
\hline $\begin{array}{l}\text { Cadmium }(\mathrm{Cd}) \\
\mathrm{ppm}\end{array}$ & $\begin{array}{c}0.035 \\
\pm 0.008\end{array}$ & $\begin{array}{c}0.062 \\
\pm 0.017\end{array}$ & $\begin{array}{c}0.037 \\
\pm 0.013\end{array}$ & $\begin{array}{c}0.036 \\
\pm 0.007\end{array}$ & $\begin{array}{c}0.145 \\
\pm 0.023^{*}\end{array}$ & $\begin{array}{c}0.172 \\
\pm 0.022^{* * * *}\end{array}$ & $\begin{array}{c}0.140 \\
\pm 0.019^{*}\end{array}$ & $\begin{array}{c}0.115 \\
\pm 0.012\end{array}$ \\
\hline Lead $(\mathrm{Pb}) \mathrm{ppm}$ & $\begin{array}{c}0.027 \\
\pm 0.004\end{array}$ & $\begin{array}{c}0.038 \\
\pm 0.003\end{array}$ & $\begin{array}{c}0.033 \\
\pm 0.005\end{array}$ & $\begin{array}{c}0.029 \\
\pm 0.007\end{array}$ & $\begin{array}{c}1.560 \\
\pm 0.047^{* * * *}\end{array}$ & $\begin{array}{c}1.780 \\
\pm 0.038^{* * *}\end{array}$ & $\begin{array}{c}1.260 \\
\pm 0.056^{* *}\end{array}$ & $\begin{array}{c}1.320 \\
\pm 0.037^{* * *}\end{array}$ \\
\hline $\begin{array}{l}\text { Copper }(\mathrm{Cu}) \\
\text { ppm }\end{array}$ & $\begin{array}{c}0.42 \\
\pm 0.019\end{array}$ & $\begin{array}{c}0.380 \\
\pm 0.024\end{array}$ & $\begin{array}{l}0.0270 \\
\pm 0.007\end{array}$ & $\begin{array}{c}0.038 \\
\pm 0.006\end{array}$ & $\begin{array}{c}2.648 \\
\pm 0.089^{* * *}\end{array}$ & $\begin{array}{c}0.962 \\
\pm 0.34^{* * *}\end{array}$ & $\begin{array}{c}0.994 \\
\pm 0.110^{* * * *}\end{array}$ & $\begin{array}{c}0.984 \\
\pm 0.063^{* * *}\end{array}$ \\
\hline
\end{tabular}

Permissible limit of heavy metals in organs of fish is according to WHO, 1992, Cadmium is 0.05ppm, Lead is and Copper is 0.5ppm. 
Table 3: Mean level of heavy metal concentration in some organs of Nile Tilapia collected from El-Tawilla drainage comparing with samples collected from River Nile Mansoura.

\begin{tabular}{|c|c|c|c|c|c|c|c|c|}
\hline \multirow{2}{*}{$\begin{array}{l}\text { organ } \\
\text { heavy metal }\end{array}$} & \multicolumn{4}{|c|}{$\begin{array}{c}\text { Samples of fish organs collected from } \\
\text { River Nile Mansoura }\end{array}$} & \multicolumn{4}{|c|}{$\begin{array}{c}\text { Samples of fish organs collected from } \\
\text { El-Tawilla drainage }\end{array}$} \\
\hline & Muscle & Liver & Kidney & Gills & Muscle & Liver & Kidney & Gills \\
\hline \multirow{2}{*}{$\begin{array}{c}\text { Cadmium }(\mathrm{Cd}) \\
\text { ppm }\end{array}$} & 0.035 & 0.062 & 0.037 & 0.036 & 0.113 & 0.132 & 0.098 & 0.088 \\
\hline & \pm 0.008 & \pm 0.017 & \pm 0.013 & \pm 0.007 & $\pm 0.026^{* * *}$ & $\pm 0.012^{* *}$ & $\pm 0.012^{* * * *}$ & \pm 0.016 \\
\hline \multirow{2}{*}{ Lead $(\mathrm{Pb}) \mathrm{ppm}$} & 0.027 & 0.038 & 0.033 & 0.029 & 0.875 & 0.925 & 0.765 & 0.935 \\
\hline & \pm 0.004 & \pm 0.003 & \pm 0.005 & \pm 0.007 & $\pm 0.07^{* * *}$ & $\pm 0.023^{* * * *}$ & $\pm 0.011^{* * * *}$ & $\pm 0.027^{* * *}$ \\
\hline \multirow{2}{*}{$\begin{array}{c}\text { Copper }(\mathrm{Cu}) \\
\text { ppm }\end{array}$} & 0.42 & 0.380 & 0.0270 & 0.039 & 0.788 & 0.621 & 0.667 & 0.598 \\
\hline & \pm 0.019 & \pm 0.024 & \pm 0.007 & \pm 0.006 & $\pm 0.032^{* * * *}$ & $\pm 0.06^{* *}$ & $\pm 0.014^{* * * *}$ & $\pm 0.029^{* * * *}$ \\
\hline
\end{tabular}

Permissible limit of heavy metals in organs of fish is according to WHO, 1992, Cadmium is 0.05ppm, Lead is 0.5ppm and Copper is $0.5 \mathrm{ppm}$.

Table 4: Mean level of heavy metal concentration in some organs of Nile Tilapia collected from Battra drainage comparing with samples collected from River Nile Mansoura.

\begin{tabular}{|c|c|c|c|c|c|c|c|c|}
\hline \multirow{2}{*}{$\begin{array}{l}\text { organ } \\
\text { heavy metal }\end{array}$} & \multicolumn{4}{|c|}{$\begin{array}{c}\text { Samples of fish organs collected from } \\
\text { River Nile Mansoura }\end{array}$} & \multicolumn{4}{|c|}{$\begin{array}{c}\text { Samples of fish organs collected from } \\
\text { Battra drainage }\end{array}$} \\
\hline & Muscle & Liver & Kidney & Gills & Muscle & Liver & Kidney & Gills \\
\hline \multirow{2}{*}{$\begin{array}{c}\text { Cadmium }(\mathrm{Cd}) \\
\text { ppm }\end{array}$} & 0.035 & 0.062 & 0.037 & 0.036 & 0.103 & 0.121 & 0.092 & 0.83 \\
\hline & \pm 0.008 & \pm 0.017 & \pm 0.013 & \pm 0.007 & $\pm 0.012^{* * * *}$ & \pm 0.018 & \pm 0.009 & \pm 0.008 \\
\hline \multirow{2}{*}{ Lead $(\mathrm{Pb}) \mathrm{ppm}$} & 0.027 & 0.038 & 0.033 & 0.029 & 0.715 & 0.788 & 0.652 & 0.702 \\
\hline & \pm 0.004 & \pm 0.003 & \pm 0.005 & \pm 0.007 & $\pm 0.016^{* * *}$ & $\pm 0.020^{* * *}$ & $\pm 0.018^{* * *}$ & $\pm 0.012^{* * *}$ \\
\hline \multirow{2}{*}{$\begin{array}{l}\text { Copper }(\mathrm{Cu}) \\
\text { ppm }\end{array}$} & 0.42 & 0.380 & 0.0270 & 0.039 & $0.667^{* *}$ & $0.595^{* *}$ & $0.625^{* *}$ & 0.0582 \\
\hline & \pm 0.019 & \pm 0.024 & \pm 0.007 & \pm 0.006 & \pm 0.023 & \pm 0.014 & \pm 0.018 & \pm 0.004 \\
\hline
\end{tabular}

Permissible limit of heavy metals in organs of fish is according to WHO, 1992, Cadmium is 0.05ppm, Lead is $0.5 \mathrm{ppm}$ and Copper is $0.5 \mathrm{ppm}$. 
Table 5: Mean level of heavy metal concentration in some organs of Nile Tilapia collected from River Nile Damietta town comparing with samples collected from River Nile Mansoura.

\begin{tabular}{|c|c|c|c|c|c|c|c|c|}
\hline \multirow{2}{*}{ organ } & \multicolumn{3}{|c|}{$\begin{array}{r}\text { Samples of fish organs collected } \\
\text { from River Nile Mansoura }\end{array}$} & \multicolumn{3}{c|}{$\begin{array}{r}\text { Samples of fish organs collected from } \\
\text { River Nile Damietta }\end{array}$} \\
\cline { 2 - 9 } heavy metal & Muscle & Liver & Kidney & Gills & Muscle & Liver & Kidney & Gills \\
\hline $\begin{array}{c}\text { Cadmium } \\
\text { (Cd) ppm }\end{array}$ & $\begin{array}{c}0.035 \\
\pm 0.008\end{array}$ & $\begin{array}{c}0.062 \\
\pm 0.017\end{array}$ & $\begin{array}{c}0.037 \\
\pm 0.013\end{array}$ & $\begin{array}{c}0.036 \\
\pm 0.007\end{array}$ & $\begin{array}{c}0.120 \\
\pm 0.013\end{array}$ & $\begin{array}{c}0.091 \\
\pm 0.010\end{array}$ & $\begin{array}{c}0.060 \\
\pm 0.015\end{array}$ & $\begin{array}{c}0.059 \\
\pm 0.008\end{array}$ \\
\hline $\begin{array}{c}\text { Lead }(\mathrm{Pb}) \\
\text { ppm }\end{array}$ & 0.027 & 0.038 & 0.033 & 0.029 & 0.654 & 0.593 & 0.515 & 0.502 \\
\pm 0.004 & \pm 0.003 & \pm 0.005 & \pm 0.007 & $\pm 0.014^{* *}$ & $\pm 0.016^{* *}$ & $\pm 0.08^{* * *}$ & $\pm 0.04^{* *}$ \\
\hline $\begin{array}{c}\text { Copper } \\
(\mathrm{Cu}) \mathrm{ppm}\end{array}$ & $\begin{array}{c}0.42 \\
\pm 0.019\end{array}$ & $\begin{array}{c}0.380 \\
\pm 0.024\end{array}$ & $\begin{array}{c}0.0270 \\
\pm 0.007\end{array}$ & $\begin{array}{c}0.039 \\
\pm 0.006\end{array}$ & $\begin{array}{c}0.635 \\
\pm 0.012^{* *}\end{array}$ & $\begin{array}{c}0.822 \\
\pm 0.013^{* * *}\end{array}$ & $\begin{array}{c}0.514 \\
\pm 0.018^{* *}\end{array}$ & $\begin{array}{c}0.576 \\
\pm 0.09^{* * *}\end{array}$ \\
\hline
\end{tabular}

Permissible limit of heavy metals in organs of fish is according to WHO, 1992, Cadmium is 0.05ppm, Lead is $0.5 \mathrm{ppm}$ and Copper is $0.5 \mathrm{ppm}$.

Table 6: Chemical constituents of water samples collected from different locations.

\begin{tabular}{|c|c|c|c|c|c|c|}
\hline Parameter & $\begin{array}{c}\text { Ammonia } \\
(\mathrm{mg} / \mathrm{l})\end{array}$ & $\begin{array}{c}\text { Nitrite } \\
(\mathrm{mg} / \mathrm{l})\end{array}$ & $\begin{array}{c}\text { Nitrate } \\
(\mathrm{mg} / \mathrm{l})\end{array}$ & $\begin{array}{c}\text { Chloride } \\
(\mathrm{mg} / \mathrm{l})\end{array}$ & $\begin{array}{c}\text { Sulphates } \\
(\mathrm{mg} / \mathrm{l})\end{array}$ & $\begin{array}{c}\text { Total } \\
\text { hardness } \\
\mathrm{CaCo}_{3} \\
\mathrm{mg} / \mathrm{l})\end{array}$ \\
\hline $\begin{array}{c}\text { Nile River } \\
\text { Mansoura }\end{array}$ & $\begin{array}{c}0.12 \\
\pm 0.02 \mathrm{e}\end{array}$ & $\begin{array}{c}0.08 \\
\pm 0.004 \mathrm{e}\end{array}$ & $\begin{array}{c}1.41 \\
\pm 0.07 \mathrm{e}\end{array}$ & $\begin{array}{c}24.22 \\
\pm 1.15 \mathrm{e}\end{array}$ & $\begin{array}{c}33.23 \\
\pm 3.21 \mathrm{e}\end{array}$ & $\begin{array}{c}50.12 \\
\pm 3.53 \mathrm{e}\end{array}$ \\
\hline $\begin{array}{c}\text { Met-Annter } \\
\text { drainage }\end{array}$ & $\begin{array}{c}1.498 \\
\pm 0.08 \mathrm{a}^{* * *}\end{array}$ & $\begin{array}{c}1.22 \\
\pm 0.05 \mathrm{a}^{* * *}\end{array}$ & $\begin{array}{c}5.56 \\
\pm 0.42 \mathrm{a}^{* * *}\end{array}$ & $\begin{array}{c}122.49 \\
\pm 9.25 \mathrm{a}^{* * *}\end{array}$ & $\begin{array}{c}312.5 \\
\pm 18.16 \mathrm{a}^{* * *}\end{array}$ & $\begin{array}{c}220.35 \\
\pm 16.22 \mathrm{a}^{* * * *}\end{array}$ \\
\hline $\begin{array}{c}\text { El-Tawilla } \\
\text { drainage }\end{array}$ & $\begin{array}{c}0.525 \\
\pm 0.06 \mathrm{~b}^{* * *}\end{array}$ & $\begin{array}{c}0.493 \\
\pm 0.05 \mathrm{~b}^{* * *}\end{array}$ & $\begin{array}{c}3.566 \\
\pm 0.83^{* *}\end{array}$ & $\begin{array}{c}88.25 \\
\pm 9.56 \mathrm{c}^{* * *}\end{array}$ & $\begin{array}{c}111.34 \\
\pm 8.25 \mathrm{~b}^{* * *}\end{array}$ & $\begin{array}{c}150.43 \\
\pm 10.55 \mathrm{~b}^{* * * *}\end{array}$ \\
\hline $\begin{array}{c}\text { Battra } \\
\text { drainage }\end{array}$ & $\begin{array}{c}0.320 \\
\pm 0.015 \mathrm{c}^{* * * *}\end{array}$ & $\begin{array}{c}0.365 \\
\pm 0.03 \mathrm{c}^{* * *}\end{array}$ & $\begin{array}{c}2.43 \\
\pm 0.62 \mathrm{c}\end{array}$ & $\begin{array}{c}95.24 \\
\pm 11.61 \mathrm{~b}^{* * *}\end{array}$ & $\begin{array}{c}99.44 \\
\pm 6.12 \mathrm{c}^{* * *}\end{array}$ & $\begin{array}{c}111.65 \\
\pm 7.88 \mathrm{c}^{* * *}\end{array}$ \\
\hline $\begin{array}{c}\text { River Nile } \\
\text { Damietta }\end{array}$ & $\begin{array}{c}0.210 \\
\pm 0.03 \mathrm{~d}^{*}\end{array}$ & $\begin{array}{c}0.148 \\
\pm 0.03 \mathrm{~d}^{* *}\end{array}$ & $\begin{array}{c}1.61 \\
\pm 0.05 \mathrm{~d}\end{array}$ & $\begin{array}{c}55.60 \\
\pm 5.23 \mathrm{~d}^{*}\end{array}$ & $\begin{array}{c}60.18 \\
\pm 6.11^{* * *}\end{array}$ & $\begin{array}{c}70.14 \\
\pm 5.72 \mathrm{~d}\end{array}$ \\
\hline
\end{tabular}

*** $\mathrm{P}<0.001 \quad * * \mathrm{P}<0.01 \quad * \mathrm{P}<0.05$

Means with the same letters in the same row are not significantly different 
Table 7: Malathion concentrations in water and fish samples from different locations.

\begin{tabular}{|c|c|c|}
\hline Malathion & $\begin{array}{c}\text { Malathion } \\
\text { in water }\end{array}$ & $\begin{array}{c}\text { Malathion } \\
\text { in fish }\end{array}$ \\
\hline Met-Annter drainage & $\begin{array}{c}0.387 \\
\pm 0.015^{* * *} \\
\pm 0.016^{* * *}\end{array}$ \\
\hline El-Tawilla drainage & $\begin{array}{c}0.472 \\
\pm 0.018^{* * *}\end{array}$ & $\begin{array}{c}0.121 \\
\pm 0.018^{* * *}\end{array}$ \\
\hline Battra drainage & 0.256 & 0.115 \\
& \pm 0.012 & $\pm 0.012^{* * *}$ \\
\hline River Nile Damietta & 0.075 & $\begin{array}{c}0.021 \\
\pm 0.005\end{array}$ \\
\hline
\end{tabular}

$* * * \mathrm{P}<0.001$

Table 8: Mean value of blood pictures of fish collected from different locations

\begin{tabular}{|c|c|c|c|c|c|}
\hline $\begin{array}{c}\text { Location } \\
\text { Blood picture }\end{array}$ & $\begin{array}{c}\text { River Nile } \\
\text { Mansoura }\end{array}$ & $\begin{array}{c}\text { Met-Annter } \\
\text { drainage }\end{array}$ & $\begin{array}{c}\text { El-Tawilla } \\
\text { drainage }\end{array}$ & $\begin{array}{c}\text { Battra } \\
\text { drainage }\end{array}$ & $\begin{array}{c}\text { River Nile } \\
\text { Damietta }\end{array}$ \\
\hline $\begin{array}{c}\text { Total RBCs } \\
\left(\mathrm{X} 10^{6} / \mu \mathrm{l}\right)\end{array}$ & $\begin{array}{c}1.87 \\
\pm 0.09 \mathrm{a}\end{array}$ & $\begin{array}{c}1.45 \\
\pm 0.08 \mathrm{e}^{* *}\end{array}$ & $\begin{array}{c}1.63 \\
\pm 0.06 \mathrm{c}^{*}\end{array}$ & $\begin{array}{c}1.58 \\
\pm 0.08 \mathrm{~d}^{* *}\end{array}$ & $\begin{array}{c}1.78 \\
\pm 0.07 \mathrm{~b}\end{array}$ \\
\hline $\begin{array}{c}\text { Haemoglobin } \\
(\mathrm{gm} / \mathrm{dl})\end{array}$ & $\begin{array}{c}7.43 \\
\pm 0.51 \mathrm{a}\end{array}$ & $\begin{array}{c}5.03 \\
\pm 0.30 \mathrm{~d}^{* *}\end{array}$ & $\begin{array}{c}5.89 \\
\pm 0.55 \mathrm{dc}^{* *}\end{array}$ & $\begin{array}{c}6.12 \\
\pm 0.48 \mathrm{c}^{*}\end{array}$ & $\begin{array}{c}6.92 \\
\pm 0.020\end{array}$ \\
\hline $\begin{array}{c}\text { Packed cell } \\
\text { volume \% }\end{array}$ & $\begin{array}{c}22.11 \\
\pm 0.86 \mathrm{a}\end{array}$ & $\begin{array}{c}16.54 \\
\pm 0.72 \mathrm{~d}^{* * *}\end{array}$ & $\begin{array}{c}16.92 \\
\pm 0.76 \mathrm{~d}^{* * *}\end{array}$ & $\begin{array}{c}18.18 \\
\pm 0.82 \mathrm{c}^{* *}\end{array}$ & $\begin{array}{c}20.21 \\
\pm 0.94 \mathrm{~b}\end{array}$ \\
\hline $\begin{array}{c}\text { Total WBCs } \\
\left(\mathrm{X} 10^{3} / \mu \mathrm{l}\right)\end{array}$ & $\begin{array}{c}29.26 \\
\pm 1.21 \mathrm{e}\end{array}$ & $\begin{array}{c}39.11 \\
\pm 0.92 \mathrm{a}^{* * *}\end{array}$ & $\begin{array}{c}38.24 \\
\pm 0.79 \mathrm{~b}^{* * *}\end{array}$ & $\begin{array}{c}36.75 \\
\pm 0.82 \mathrm{c}^{* *}\end{array}$ & $\begin{array}{c}34.12 \\
\pm 0.73 \mathrm{~d}^{*}\end{array}$ \\
\hline
\end{tabular}

$* * * \mathrm{P}<0.001 \quad * * \mathrm{P}<0.01 \quad * \mathrm{P}<0.05$

Means with the same letters in the same row are not significantly different.

Table 9: Some liver and kidney functions of fish from different locations 
Assiut Vet. Med. J. Vol. 57 No. 129 April 2011

\begin{tabular}{|c|c|c|c|c|c|}
\hline $\begin{array}{c}\text { Location } \\
\text { Serum }\end{array}$ & $\begin{array}{c}\text { River Nile } \\
\text { Mansoura }\end{array}$ & $\begin{array}{c}\text { Met-Annter } \\
\text { drainage }\end{array}$ & $\begin{array}{c}\text { El-Tawilla } \\
\text { drainage }\end{array}$ & $\begin{array}{c}\text { Battrea } \\
\text { drainage }\end{array}$ & $\begin{array}{c}\text { River Nile } \\
\text { Dameitta }\end{array}$ \\
\hline Total protein & $\begin{array}{c}4.83 \\
\pm 0.28 \mathrm{a}\end{array}$ & $\begin{array}{c}3.52 \\
\pm 0.21 \mathrm{c}^{* *}\end{array}$ & $\begin{array}{c}3.75 \\
\pm 0.32 \mathrm{c}^{* *}\end{array}$ & $\begin{array}{c}3.40 \\
\pm 0.38 \mathrm{c}^{* *}\end{array}$ & $\begin{array}{c}3.92 \\
\pm 0.43 \mathrm{~b}^{*}\end{array}$ \\
\hline ALT & $\begin{array}{c}9.12 \\
\pm 0.53 \mathrm{~d}\end{array}$ & $\begin{array}{c}13.24 \\
\pm 0.61 \mathrm{a}^{* * *}\end{array}$ & $\begin{array}{c}12.32 \\
\pm 0.41 \mathrm{~b}^{* * *}\end{array}$ & $\begin{array}{c}12.11 \\
\pm 0.58 \mathrm{~b}^{* * *}\end{array}$ & $\begin{array}{c}11.48 \\
\pm 0.43 \mathrm{c}^{* *}\end{array}$ \\
\hline AST & $\begin{array}{c}280.75 \\
\pm 1.22 \mathrm{~d}\end{array}$ & $\begin{array}{c}297.60 \\
\pm 2.62 \mathrm{a}^{* * *}\end{array}$ & $\begin{array}{c}290.84 \\
\pm 2.40 \mathrm{~b}^{* *}\end{array}$ & $\begin{array}{c}295.44 \\
\pm 2.20 \mathrm{a}^{* * *}\end{array}$ & $\begin{array}{c}288.54 \\
\pm 1.94 \mathrm{c}\end{array}$ \\
\hline Creatinin & $\begin{array}{c}1.22 \\
\pm 0.07 \mathrm{c}\end{array}$ & $\begin{array}{c}2.62 \\
\pm 0.09 \mathrm{a}^{* * *}\end{array}$ & $\begin{array}{c}2.30 \\
\pm 0.06 \mathrm{a}^{* * *}\end{array}$ & $\begin{array}{c}2.27 \\
\pm 0.04 \mathrm{a}^{* * *}\end{array}$ & $\begin{array}{c}1.68 \\
\pm 0.02 \mathrm{~b}\end{array}$ \\
\hline \multirow{2}{*}{ Blood urea } & $\begin{array}{c}3.20 \\
\pm 0.11 \mathrm{~d}\end{array}$ & $\begin{array}{c}5.75 \\
\pm 0.31 \mathrm{a}^{* * *}\end{array}$ & $\begin{array}{c}5.39 \\
\pm 0.28 \mathrm{a}^{* * *}\end{array}$ & $\begin{array}{c}5.18 \\
\pm 0.22 \mathrm{~b}^{* * *}\end{array}$ & $\begin{array}{c}4.20 \\
\pm 0.20 \mathrm{c}^{*}\end{array}$ \\
\hline
\end{tabular}

$* * * \mathrm{P}<0.001 \quad * * \mathrm{P}<0.01 \quad * \mathrm{P}<0.05$

Means with the same letters in the same row are not significantly different

Table 10: Glutathione system in hepatic tissue in fish $(\mu \mathrm{mole} / \mathrm{gm}$ protein)

\begin{tabular}{|c|c|c|c|}
\hline $\begin{array}{c}\text { Glutathione system } \\
\text { locations }\end{array}$ & Total glutathione & $\begin{array}{c}\text { Reduced } \\
\text { glutathione }\end{array}$ & Oxidized glutathion \\
\hline River Nile Mansoura & $\begin{array}{c}58.42 \\
\pm 1.62 \mathrm{a}\end{array}$ & $\begin{array}{c}53.92 \\
\pm 1.20 \mathrm{a}\end{array}$ & $\begin{array}{c}4.50 \\
\pm 0.22 \mathrm{~d}\end{array}$ \\
\hline Met-Annter drainage & $\begin{array}{c}52.62 \\
\pm 1.52^{*}\end{array}$ & $\begin{array}{c}47.73 \\
\pm 1.12 \mathrm{~d}^{* * *}\end{array}$ & $\begin{array}{c}4.89 \\
\pm 0.24 \mathrm{~b}^{*}\end{array}$ \\
\hline El-Tawilla drainage & 53.37 & 48.26 & 5.11 \\
$\pm 1.45 \mathrm{c}^{*}$ & $\pm 1.11 \mathrm{~d}^{* * *}$ & $4.28 \mathrm{a}^{* *}$ \\
\hline & 53.85 & 49.02 & 4.83 \\
Battra drainage & $\pm 1.61 \mathrm{c}^{*}$ & $\pm 1.16 \mathrm{c}^{* *}$ & $\pm 0.25 \mathrm{~b}^{*}$ \\
& 56.30 & 51.52 & 4.79 \\
& $\pm 1.74 \mathrm{~b}$ & $\pm 1.18 \mathrm{~b}$ & $\pm 0.26 \mathrm{c}$ \\
\hline
\end{tabular}

$* * * \mathrm{P}<0.001 \quad * * \mathrm{P}<0.01 \quad * \mathrm{P}<0.05$

Means with the same letters in the same row are not significantly different.

\section{DISCUSSION}


The changes in the physicochemical properties of water samples collected from the different locations reflect the differences in the effluents that discharged to each location such as Agricultural drainage, industrial effluents and waste municipal (Boyd, 1990).

$\mathrm{pH}$ values of water from different locations were in normal range. While, there was a slight increase in $\mathrm{pH}$ of water of Met-Annter drainage due to industrial, agriculture water drainage and in alkalinity was due to the uptake of free carbon dioxide from water and also precipitation of calcium carbonate (Boyd, 1990; Saeed, 2000).

Polluted water with heavy metals is generally associated with industrial, agricultural and municipal discharges (Haggag et al., 1999; Zaghloul, 2000).

In the present study the highest increasing of heavy metal concentration was in water samples collected from Met-Annter drainage because of the presence of factory of fertilization behind this drainage with the mean $0.098-0.89$ and $1.48 \mathrm{mg} / \mathrm{l}$ for cadmium, lead and copper respectively and the lowest concentration was in the River Nile with the mean $0.048,0.009$ and $0.067 \mathrm{mg} / \mathrm{l}$

For cadmium, lead and copper respectively, so the collected samples from different locations attributed to the differences in the effluents such as waste municipal industrial and agricultural drainage water). This is in agreement with Nagdi and Shaker (1998) who concluded the increasing of heavy metals in drainage water due to the decomposition of the organic matter, use of fertilizers and other chemicals in agriculture.

As heavy metals discharged into water, it enters the food chain bioaccumulation within fish tissue and so to man Ajmal et al. (1985). Meanwhile the concentration of studied heavy metals in vital organs and muscles of Nile Tilapia fish showed the highest increase in fish samples collected from Met-Annter drainage with a mean 0.145- 0.172-0.140 and $0.115 \mathrm{ppm}$ and the lowest samples from River Nile Mansoura with the mean0.035 - $0.062-0.037$ and $0.036 \mathrm{ppm}$ for cadmium, lead and copper respectively as shown in Table 2. This agreed with Zaghloul, (2000).

The highest increase of Cadmium, Lead and Copper in muscle and liver samples were collected from Met - Annter drainage with the means $0.1450 .1721 .560-1.7802 .648$ and $0.962 \mathrm{PPm}$ respectively, while the lowest concentration in muscle and gills of samples collected from Nile River Mansoura with the means ( 0.035 - 0.036- 0.027 $0.029-0.42$ and 0.038 ) respectively as shown in table 2 Heavy metals 
were significantly higher in liver tissue recorded before by Shereif and Moaty, (1995). The highest concentration of studied heavy metal in liver tissue may be due to the major role of liver in the detoxification and protection from heavy metal (Pratap et al., 1989).

In this study, the highest ammonia concentration was in samples collected from Met-Annter drainage and having ammonia concentration above recommended by WHO (1993). These are in agreement with Mohamed (2005) who recorded the increase in ammonia concentration in water samples collected from Abu-Za'baal ponds. The lowest ammonia concentration were found in water samples collected from River Nile in Mansoura. The ammonia concentration was found within permissible limits according WHO (1992).

Samples collected from River Nile Damietta having higher ammonia concentration than River Nile in Mansoura that above the permissible limit of ammonia according to WHO (1992). Water samples from drainages have highest concentration of ammonia because ammonia present in high concentration in regions of intensive agricultural activities fertilizer runoff and septic tank which discharges from the soil to the drainages (Yong and Cheng, 2007).

Nitrite concentrations within samples collected from Met-Annter drainage is the highest which agree with Akoto and Adiyiah (2007), while lowest nitrite concentration were found in samples collected from River Nile in Mansoura and these are in agreement with Okafor and Ogbanna (2003).

Regarding nitrate concentration, the highest concentration was found in water samples collected from drainages while the lowest concentration in water sample collected from River Nile. These are in agreement with Shaaban et al. (1993); Samaha (1985).

Concerning chloride concentration all collected samples are within permissible limit of chloride concentration but drainage have higher concentration than River Nile according to (WHO, 1992) these results are in agreement with Wakawa et al. (2008).

Regarding sulphate concentration in water, samples collected from Met-Annter drainage showed the highest concentration. These results are in agreement with Dange et al. (2005).

The lowest concentration of sulphate was found in water samples collected from River Nile Mansoura and these are in agreement with Wakawa et al. (2008).

Total hardness in water samples collected from all drainages were higher than that collected from River Nile as shown in table 6, and 
these are in agreement with Shaaban et al. (1993). The increasing of total hardness in drainages is due to agriculture drainage water and changes in alkalinity (Boyd, 1990; Saeed, 2000).

In present study malthion was detected in all drainages but not found in River Nile Mansoura. The highest malathion concentrationwas in El-Tawilla drainage. Presence of malathion in drainages and not found in River Nile indicates a runoff of the pesticide from the agricultural land to the drainage canals. Hydrolysis is a major pathway of degradation of malathion in aquatic systems and increase with increasing alkalinity and temperature Malathion concentration were detected in fish samples collected from El-Tawilla drainage Malathion concentration in water was higher than in fish samples as shown in Table 7, (Guerrant et al., 1970).

The lowest total protein concentration in serum samples were recorded in fish samples collected from Met-Anntar drainage compared with samples collected from River Nile and other location.

Total protein in serum samples collected from Met-Annter drainage showed the highest decreasing compared with samples collected from River Nile and samples from other locations. This decrease may be due to liver damage. These results were agreed with Abd-Alla et al. (2002).

A significant increase in AST/ALT activity in; Met-Annter, ElTawilla and Battra drainage were recorded. The increase of liver enzymatic activity may be due to hepatic damage resulted from oxidative stress in fish exposed to pollution. This is in agreeing with Hema et al. (1987); Zikic et al. (2001). Concerning kidney function, it showed an increase in serum creatinine and blood urea, these agree with Hafez et al. (2003) who reported an increases in blood urea and serum creatinine in fish collected from polluted locations due to kidney damage.

Haematological studies were carried out for evaluation blood parameters to investigate physiological changes caused by environmental pollutants (Ghazaly and Said, 1995; El-Naggar et al., 1998; Haggag et al., 1999).

The haematological analysis of blood samples showed significant decrease in RBCs, $\mathrm{Hb}$ and PCV valued. Reduction in red blood corpuscles production in the haematopoietic organ under the action of heavy metals accumulated at high concentrations in tissue and due to intrahepatic and intrasplenic haemmorrhage. 
Decrease in RBCs may be due to reduction in red blood corpuscles production in the bone marrow under the action of heavy metals. These results agreed with Abbas (1994); Marie (1990). Leucocytosis showed significant increase, which, may be due to release of corticoids into circulation under the effect of heavy metal and may be due to hepatitis and nephrites under the effect of heavy metal accumulated in liver and kidney. These are in agreement with Hafez et al. (2003).

In present study there was a decrease in hepatic reduced glutathione and total glutathione mean while there was an increase in oxidized glutathione. In fact depletion of liver glutathione often occurs in liver injuries (Uhling and Wendel, 1992).

\section{CONCLUSION}

The increasing oxidized glutathione revealed a negative shifting of GSH/GSSII, which in turn should be a strong evidence of liver injury. All these pollutants mentioned before, resulted in a strong metabolic effect shifting of the glutathione system to the oxidized side that causing hepatotoxic effect in fish.

\section{REFERENCES}

Abbas, H.H. (1994): Effect of lead on some physiological and biochemical aspect of Nile Tilapia; Oreochromis niloticus. M.Sc.Thesis faculty of Science, Cairo University, Egypt.

Abd El-Hamid, A.M.; Maha, M.M.; Gawish, A.N. and Soryal, K.A., (2006): Comparative study between desert cultivated and natural fisheries of Mullet in Egypt, concerning heavy metals. J. Agric. Sci, Mansoura Univ., 31(9): 5665-5680.

Abd-Allah, G.A.; Mansy, S.E.; Khider, A.A.; Hassan, S.H. and ElFayoumi, R.I. (2002): Evaluation of physiological changes in canal cat fish during exposure to different levels of metal pollution Proc $2^{\text {nd }}$ Conf. Food Borne Contamination and Egyptians Health 23-24 April, El-Mansoura, Egypt. P.201-211.

Ajmal, M.; Khan, M.A. and Nomani, A.A. (1985): Distribution of heavy metals in plant and fish of the Yamuna River (India). Environ. Monit. Assess. (5): 361-367. 
Akoto, O. and Adiyiah, J. (2007): Chemical analysis of drinking water from some communities in the Brong Ahafo region. Int. J. Environ. Sci. Tech. 4(2): 211-213.

Anderson, M.E. (1985): Determination of glutathione and glutathione disulphide in biological samples. Method Enzymol .113: 548-551.

APHA, (1960): Standard methods for the examination of water and waste water $11^{\text {th }}$ Ed. Washington, D.C

APHA, (1985): Standard methods for the examination of water and waste water $16^{\text {th }}$ Ed. Washington, D.C

APHA, (1998): Standard methods for the examination of water and waste water, $20^{\text {th }}$ Ed. Washington, D.C

Aspelin, A.L. (1998): USEPA Office of Pesticide Programs Pesticide Industry Sales and Usage 1996 and 1997 Market Estimates. http://www.epa.gov/oppbeadl/pestsales/97.pestsales/intro.htm\# overview

Boyd, C.E. (1990): Water quality in pound for aquaculture in warm water fish ponds. Aubmn Univ., Alabama, U.S.A.

Burger, J.; Stern, A.H. and Gochfeld, M. (2005): Mercury in Commercial Fishoptimizing individual choices to Reduce Risk. Environ. Health Pprespect. 113(3): 266-271.

Calderon, R.L. (2000): The Epidemiology of Chemical Contaminants of Drinking Water. Food and Chem. Toxicol. (38), 513-520.

Capar, M.S.G. (1977): Metals and other elements. J. A.O.A.C., 60. 1401-1408.

Dange, D.H.; William, E.O. and Paul, B.T. (2005): Comparative assessment of the physico-chemical and bacteriological qualities of selected streams in Louisiana. Int. J. Environ. Res. Public. Health. 2(1): 94-100.

Darwish, A.M.; El-Mossalami, M.K. and El-Bassouny, R.A. (2003): Quality assurance of some fatty fishes. Assiut Vet. M. J. 49 (98): 79-96.

De Gregori, L.; Pinochet, H.; Delgado, D.; Gras, N. and Munoz, L. (1994): Heavy metals in Bivalve muscles and their habitats from different sites along the children coast. Bull. Environ. Contam. Toxicol., 52: 261-268.

Denslow, N.D.; Garcia-Reyero, N. and Barber, D.S. (2007): Fish. Chips. The use of micro arrays for aquatic toxicology. Mol. Biosyst. 3(3):172-177. 
Doumas, B.; Bayso, D.; Crter R., Peters T. and Schaffer R., (1981):Determination of serum total protein. Clin. Chem. 27: 1642.

El-Naggar, G.O.; Zaghloul, K.H.; Salah El-Deen, M.A. and Abo-Hegab, $S$. (1998): Studies on the effects of industrial water pollution along different sites of the River Nile on some physiological parameters of Nile Tilapia; Oreochromis niloticus, $4^{\text {th }}$ Vet. Med. Zag. Congress (26-28 August, 1998 hn Hurghada): 713-735.

Fawell, J. and Nieuwenhuijsen, M.J. (2003): Contaminants in drinking water. Environmental pollution and health. British Medical Bulletin.68: 199-208.

Ghazally, K.S. and Said, K.M. (1995): Physiological characteristics of Tilapia nilotica under acute stress of copper. J. Egypt. Ger. Soc. Zool. (16A): 287-301.

Gold FranK, L.; Fomenbaum, N.; Lewin, N; Weisman, R. and Hawland, M. (2001): Gold Frank's. Toxicological Emergrnices $5^{\text {th }}$ Ed. Prentic- Hall International INC. New Jersey USA.

Greig, R.A.; Sawyer, T.K.; Lewis, E.T. and Galasso, M.E. (1982): A study of metal concentrations in relation to gill color and pathology in rock carb. Arch. Environ. Contam. Toxicol. (8): 589-597.

Guerrant, G.O.; Fetzer, Jr.L.E. and Miles, J.W. (1970): Pesticide residues in Hale Country. Texas, before and after ultra low volume aerial application of malathion. Pesticides Monitoring J. 4(16): 14-20.

HACH, (2003): HACH water analysis Handbook. Printed in U.S.A.

Hafez, M.H.; Anisa, M.; Moustafa, Aly S.M.; El-Matbuli, M.A. and Nouh, W. (2003): Pathological and electron microscopic evaluation of the effect of water pollution with lead acetate in the health status and immune response of cat fish; Claias gariepinus. SCVMJ. VI(1) 2003.

Haggag, A.M.; Mohamed, A.S.M. and Khaled, H.Z. (1999): Seasonal effect of the industrial effluents on the Nile cat fish, Claias gariepinus. J.Egy.Ger. Soc. Zool. 28A: 365-391.

Heckman, M. (1970): Collaborative study of copper, sodium in feed by Atomic Absorption spectrophotometer and sodium and potassium by Flame Emission Spectrophotometer J. AOAC, (53): 923. 
Hema, T.; Gill, T.S. and Panda, J. (1987): Impact of chronic lead poisoning on the hematological profiles of fish. Bull. Environ. Contam. Toxicol. 38: 748.

Husden, H. and Ropaport, A. (1968): Estimation of creatinine. Clin. Chemistry 14: 222.

Jadhav, R.K.; Sharma, V.K.; Rao, G.J; Saraf, A.K. and Chandra, H. (1992): Distribution of malathion in body tissues and fluids. Forensic Science International, 52(2): 223-229.

Jehan R. Daoud; Aida M. Amin and Menha M. Abd El-Khalek, (1999): Residual analysis of some heavy metals in water and Oreochromis niloticus fish from polluted areas. Vet. Med. J. Giza 47 (3): 351-365.

Kirkwood, B.R. (1989): Essentials of medical statistics, Blackwell Scientific Publication $2^{\text {nd }}$ P. 151 .

Marie, M.A.S. (1990): Effect of sublethal concentration of zinc on blood parameters of the Nile cat fish; Clarias lazera. Proc. Zool. Soc. A.R.E. 18: 413-421.

Moalla, S.N. and Pulford, I.D. (1995): Mobility of metals in Egyptian desert soils subject to inundation by Lake Nasser. Soil Use and Management. Volume II Issue 2 page 94-98, June.

Mohamed, H.A. (2005): Physico-chemical characteristics of AbuZa'baal Ponds Egypt. Egypt. J. Aquatic Res. 31(2): 1-15.

Mona O. Abou El-Nile and Sawsan M. Arafa, (2004): Incidence of heavy metals in local and imported fish. Issn 110-2047. Alex, J.Vet. Science, 20 (1): 7-19.

Nagdi, A.Z. and Shaker, I. (1998): Effect of water sources on some microelements in fish farm. Egyptt. J. Agric. Res. 76 (1): 351-357.

Okafor, P.N. and Ogbonna, U.I. (2003): Nitrite contamination of water sources and fruit juices marketed in South-Eastern Nigeria. J. Food Comp. \& Analysis. 16(2): 213-218.

Patterson, J. (2002): Indirecttion-Comparativ dietary risk balance. The risk and benefits of fish consumption. Comments Toxicol, 8(4-6): 337-344.

Patton, C.J. and Crouch, S.R. (1977): Determination of urea. Anal. Chem 49: 464-469.

Polpraset, C. (1982): Heavy metal pollution in the Chaepharaya River, Estuary Thailand Water, Res. (16): 775-784. 
Pratap, H.B.; Lock, R.A.C. and Bonga, S.E.W. (1989): Effect of water borne and dietary Cadmium on plasma ions of the telost Oreochromis mossambicus in relation to water cacium levels. Aech, Environ. Contam. Toxicol. 18 (4): 568-575.

Reitman, A. and Frankel, S. (1957): Calorimetric method for determination of GOT and GPT. J. Clin. Path, 28: 56.

Repetto, R. and Baliga, S. (1996): Pesticides and the Immune system. The Public Health Risk. World Res. Ources Institute.

Richardson, R.J. and Murphy, S.D. (1987): Effect of glutathione depletion of tissue deposition of methyl mercury in rats. Toxicol. Appopharmacol. 31: 505-519.

Saeed, S.M. (2000): A study on factors affecting fish production from certain fish farms in the delta MSc. Thesis. Department of Biological and physical sciences Institute of Environmental studies and Research, Ain Shams University.

Samaha, H.T. (1985): The hygienic quality of some well water samples in Behera and Alexandria Governorates. Alex. J. Vet. Sci. 1(2): 236-252.

Shaaban, D.S.A.; Soliman, A.I. and Deyab, M.A. (1993): Environmental characteristic and nutrients distribution in Damietta estuary of the River Nile. J. Environ. Sci. Mansoura Univ. 6: 159-176.

Shereif, M.M. and Moaty, M.A. (1995): Concentration levels and accumulation of heavy metals in Nile Tilapia grown in wastewater-fed fish ponds. Sci. Midd. East Conf. Waste. Mang. Cairo, 284-298.

Shibamoto, T. and Bjeldanes, LF. (1993): Introduction to food toxicology Academic press Inc Harcourt Brace and company NewYork Food Science and Technology International series.

Sprenger, M.D.; Mclntosh, A.W. and Lewis, T. (1987): Variability in concentrations of selected trace elements in water and sediments of six acidic lakes. Arch. Environ. Contam. Toxicol. (16): 383-390.

Stoskopf, M.K. (1993): Fish medicine W.B. Saunders Camp. Philadelphia, PP. 113-131.

Uhling, S. and Wendel, A. (1992): The physiological consequences of glutathione variations Life Sci. 51: 1083-1094.

USEPA: office of Pesticide Programs, (2000): Overview of Malathion Risk Assessment.

http://www.epa.gov/pesticides/op/malathion/overview.htm 
Wakawa, R.J.; Uzairu, A.; Kagbu, J.A. and Balarabe, M.l. (2008): Impact assessment of effluent discharge on physiocochemical parameters and some heavy metal concentrations in surface water of river Challawa Kano Nigeria. Afr. J. Pure. Appl. Chem. 2(10): 100-106.

WHO (1992): Enviromental Health Criteria No. 134. WHO Geneva.

WHO (1993): Guide lines for Drinking - Water Quality. The International Standard Setting and Ddrinking- Water Safety. WHO Geneva.

Yang, H. and Cheng, H. (2007): Controlling nitrite level in drinking water by chlorination and chloramination. Separation and Purification Tech. 56: 392-396.

Young, D.S. (2001): Effects of diseases on clinical lab. Tests $4^{\text {th }}$ Ed. AACC.

Zaghloul, K.H. (2000): Effect of different water sources on some biological biochemical aspects of the Nile Tilapia; Oreochromis niloticus and Nile cat fish; Claias gaiepinus. Egypt. J., (34): 353-377.

Zikic, R.V.; Stajan, A.S.; Pavlovic, S.Z.; Ognjanovic, B.L. and Saicic, Z.S. (2001): Activities of superoxide dismutase and catalase in erythrocytes and plasma transaminases of gold fish (Carassius auratus gibelio Bloch.) exposed to cadmium. Physiol. Res., 5 (1): 105-111. 